Research Article

\title{
Estimation for Weibull Parameters with Generalized Progressive Hybrid Censored Data
}

\author{
Xuanjia Zuo $\mathbb{D}^{1},{ }^{1}$ Liang Wang $\mathbb{D},{ }^{1}$ Huizhong Lin $\mathbb{D}^{1},{ }^{1}$ Sanku Dey $\mathbb{D}^{2},{ }^{2}$ and Li Yan $\mathbb{D}^{3}$ \\ ${ }^{1}$ School of Mathematics, Yunnan Normal University, Kunming, China \\ ${ }^{2}$ Department of Statistics, St. Anthony's College, Shillong, India \\ ${ }^{3}$ Department of Administrative Sciences, University of Quebec in Outaouais, Gatineau, Canada \\ Correspondence should be addressed to Liang Wang; liang610112@163.com
}

Received 26 February 2021; Accepted 12 November 2021; Published 3 December 2021

Academic Editor: Feng Feng

Copyright ( $\odot 2021$ Xuanjia Zuo et al. This is an open access article distributed under the Creative Commons Attribution License, which permits unrestricted use, distribution, and reproduction in any medium, provided the original work is properly cited.

In this paper, the interest is in estimating the Weibull products when the available data is obtained via generalized progressive hybrid censoring. The testing scheme conducts products of interest under a more flexible way and allows collecting failure data in efficient and adaptable experimental scenarios than traditional lifetime testing. When the latent lifetime of products follows Weibull distribution, classical and Bayesian inferences are considered for unknown parameters. The existence and uniqueness of maximum likelihood estimates are established, and approximate confidence intervals are also constructed via asymptotic theory. Bayes point estimates as well as the credible intervals of the parameters are obtained, and correspondingly, Monte Carlo sampling technique is also provided for complex posterior computation. Extensive numerical analysis is carried out, and the results show that the generalized progressive hybrid censoring is an adaptive procedure in practical lifetime experiment, both proposed classical and Bayesian inferential approaches perform satisfactorily, and the Bayesian results are superior to conventional likelihood estimates.

\section{Introduction}

In lifetime study and reliability, due to many practical reasons such as time constraint and cost reduction, the experiment cannot be finished with all test unit failures; therefore, censoring appears as a common phenomenon in many life test studies. Generally speaking, there is only a portion of the testing units of which the exact failure times are known when censoring is involved under study. In practice, there are several types of schemes (CSs), and the most used ones are Type-I censoring and Type-II censoring, where experiments are terminated after a prefixed inspecting time or a predetermined number of failures. The conventional CSs, however, do not allow engineers to remove survival units from the experiment at points other than the final termination time. For this reason, a progressive censoring scheme was further introduced in practice, where engineers could remove units from testing at various stages. Like conventional CSs, progressive censoring also includes progressive Type-I censoring and progressive Type-II censoring. Compared to Type-I/-II censoring, it is seen that the progressive CSs are more flexible and efficient in lifetime study, and extensive works have been done on these directions. See, for example, some recent contributions of Yan et al. [1], Laumen and Cramer [2], Gunasekera [3], and Maurya and Tripathi [4] as well as the reference therein. For an exhaustive list of references and details on progressive censoring, one can refer to the monographs of Balakrishnan and Cramer [5].

With the improvement in manufacturing design and technology, modern products feature high reliability and long span; under such situations, there may not be enough failure samples or the time of the experiment could be very long in both conventional and progressive censoring. To overcome such problems, hybrid censoring schemes were further introduced in practice, which can be viewed as a mixture of Type-I and Type-II censoring in conventional and progressive censoring procedures, respectively. For 
instance, Prajapati et al. [6] and Algarni et al. [7] discussed inference based on Type-I and Type-II hybrid censoring, and Basu et al. [8], Gorny and Cramer [9], and Singh et al. [10] studied progressive hybrid censored data under different cases. For an exhaustive amount of references and further details on hybrid censoring, one can see the recent review paper [11] and the monograph of Balakrishnan and Cramer [5] within related sections. Recently, Cho et al. [12] proposed a generalized progressive hybrid censoring scheme (GPHCS) where an adequate number of observations are guaranteed within a reasonable experimental duration which in turn may yield improved estimation results, and the detailed procedure of such test can be described as follows: suppose that $n$ independent items are put in a test. Furthermore, assume that $k$ and $m$ are prefixed constants satisfying $1 \leq k<m \leq n, T$ is also prespecified monitoring time, and $r_{i} \geq 0, i=1, \ldots, m$ is prefixed censoring schemes with $\sum_{i=1}^{m} r_{i}+m=n$. Following the similar way as progressive censoring, at time of $i$ th failure $X_{i: m: n}$, the $r_{i}$ number of live units is withdrawn from the experiment at random. In this case, the test stops at point $T^{*}=\max \left\{X_{k: m: n}, \min \left\{T, X_{m: m: n}\right\}\right\}$, i.e.,

$$
T^{*}= \begin{cases}X_{k: m: n}, & \text { if } T<X_{k: m: n}<X_{m: m: n} \\ T, & \text { if } X_{k: m: n}<T<X_{m: m: n} \\ X_{m: m: n}, & \text { if } X_{k: m: n}<X_{m: m: n}<T\end{cases}
$$

Moreover, under GPHCS, one has the following three types of observations:

$$
\begin{aligned}
\text { case I }: X_{1: m: n}, X_{2: m: n}, \ldots, X_{k: m: n}, & \text { if } T<X_{k: m: n}<X_{m: m: n}, \\
\text { case II }: X_{1: m: n}, \ldots, X_{k: m: n}, \ldots, X_{d: m: n}, & \text { if } X_{k: m: n}<T<X_{m: m: n}, \\
\text { case III }: X_{1: m: n}, \ldots, X_{k: m: n}, \ldots, X_{m: m: n}, & \text { if } X_{k: m: n}<X_{m: m: n}<T,
\end{aligned}
$$

where for case II, $X_{d: m: n}<T<X_{d+1: m: n}$. A representation of GPHCS is shown in Figure 1, where $r_{k}^{*}=m-k+\sum_{i=k}^{m} r_{i}$ and $r_{d+1}^{*}=m-1+\sum_{i=d+1}^{m} r_{i}$. Therefore, from the test procedure of GPHCS, it is noted that if inadequate observations are recorded, then the test duration can be extended beyond $T$ for obtaining more test information. Moreover, it is also observed that some of the well-known censoring methods including progressive and progressive hybrid censoring are specific cases of GPHCS.

Denote $f(\cdot)$ and $S(\cdot)$ are the PDF and survival functions of the latent failure time $X$, respectively. Based on (2), the joint density function can be written as

$$
L(\text { data })= \begin{cases}c_{1} \prod_{i=1}^{k} f\left(x_{i: m: n}\right)\left[1-F\left(x_{i: m: n}\right)\right]^{r_{i}}, & \text { case I, } \\ c_{2} \prod_{i=1}^{d} f\left(x_{i: m: n}\right)\left[1-F\left(x_{i: m: n}\right)\right]^{r_{i}}[S(T)]^{r_{d+1}^{*},} & \text { case II, } \\ c_{3} \prod_{i=1}^{m} f\left(x_{i: m: n}\right)\left[1-F\left(x_{i: m: n}\right)\right]^{r_{i}}, & \text { case III, }\end{cases}
$$

where $r_{d+1}^{*}=n-\sum_{i=1}^{d}\left(r_{i}+1\right), r_{k}=n-\sum_{i=1}^{k-1}\left(r_{i}+1\right)-1$ in case I, and

$$
\begin{cases}c_{1}=\prod_{j=1}^{k} \sum_{k=j}^{m}\left(r_{k}+1\right), & \text { if } T<X_{k: m: n}<X_{m: m: n} \\ c_{2}=\prod_{j=1}^{d} \sum_{k=j}^{m}\left(r_{k}+1\right), & \text { if } X_{k: m: n}<T<X_{m: m: n} \\ c_{3}=\prod_{j=1}^{m} \sum_{k=j}^{m}\left(r_{k}+1\right), & \text { if } X_{k: m: n}<X_{m: m: n}<T .\end{cases}
$$

In this paper, we assume that the latent failure time of units follows a Weibull distribution with shape parameter $\alpha>0$ and scale parameter $\beta>0$, of which the probability density function (PDF) and the cumulative distribution function (CDF) can be expressed as

$$
\begin{aligned}
& f(x ; \alpha, \beta)=\alpha \beta x^{\alpha-1} e^{-\beta x^{\alpha}}, \\
& F(x ; \alpha, \beta)=1-e^{-\beta x^{\alpha}}, \quad x>0 .
\end{aligned}
$$

Because of its versatility in fitting time-to-failure distributions of a rather extensive variety of complex mechanisms, the Weibull distribution is one of the most popular distributions used in statistical inference of reliability, lifetime study, and survival analysis. Various problems associated with this distribution have been discussed by numerous authors. See, for example, the works of Almetwally and Almongy [13], Chen et al. [14], and Kumar and Ram [15] as well as the reference therein. 


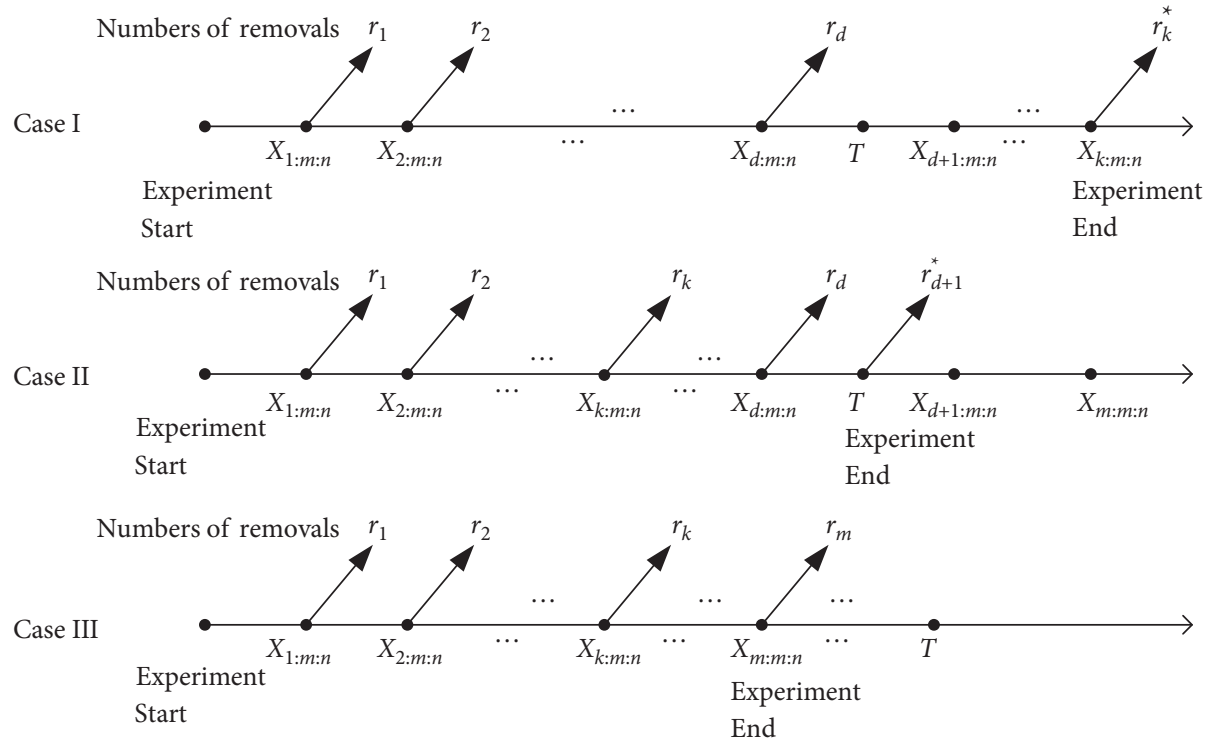

Figure 1: A sketch of the generalized progressive hybrid censoring scheme.

Suppose latent failure time (2) of units of sample size $n$ follows Weibull distribution (5) with parameters $\alpha>0$ and $\beta>0$; the likelihood function of $\alpha$ and $\beta$, namely, $L(\alpha, \beta ;$ data $)$, can be expressed as

$$
L(\alpha, \beta ; \text { data })= \begin{cases}c_{1} \prod_{i=1}^{k} \alpha \beta x_{i: m: n}^{\alpha-1} e^{-\beta x_{i: m: n}^{\alpha}\left[e^{-\beta x_{i: m: n}^{\alpha}}\right]^{r_{i}},} & \text { if } T<x_{k: m: n}<x_{m: m: n} \\ c_{2} \prod_{i=1}^{d} \alpha \beta x_{i: m: n}^{\alpha-1} e^{-\beta x_{i: m: n}^{\alpha}}\left[e^{-\beta x_{i: m: n}^{\alpha}}\right]^{r_{i}}\left[e^{-\beta T^{\alpha}}\right]^{r_{d+1}^{*}}, & \text { if } x_{k: m: n}<T<x_{m: m: n} \\ c_{3} \prod_{i=1}^{m} \alpha \beta x_{i: m: n}^{\alpha-1} e^{-\beta x_{i: m: n}^{\alpha}}\left[e^{-\beta x_{i: m: n}^{\alpha}}\right]^{r_{i}}, & \text { if } x_{k: m: n}<x_{m: m: n}<T\end{cases}
$$

Motivated by such reasons as mentioned above and due to the extensive applicability and practical significance of Weibull lifetime distribution in many real-life studies, we discussed the statistical inference for the generalized progressive hybrid censoring when the latent failure data follows the Weibull model. Our objectives in this paper are as follows: First, we estimate the parameters of the Weibull distribution under generalized progressive hybrid censoring using classical estimation methods, namely, maximum likelihood estimation (MLE). In addition, we have obtained approximate confidence intervals (ACIs) based on the asymptotic distribution of MLE. The performance of the associated estimates are demonstrated in terms of their mean squared error and root absolute bias for different sample sizes through a simulation study. The second objective is to obtain Bayes estimates based on likelihood function based on independent gamma priors. Since Bayes estimates cannot be obtained in explicit forms, then Markov Chain Monte Carlo (MCMC) sampling is used to compute the complex posterior functions and in turn to calculate Bayes estimates as well as associated highest posterior density (HPD) credible intervals. Besides, one real-life data set is analyzed to illustrate the applicability of the proposed methods.

The rest of this paper is organized as follows: Classic and Bayes estimates are presented in Sections 2 and 3, respectively. Section 4 provides numerical illustrations, and some concluding remarks are given in Section 5.

\section{Frequentist Inference}

MLEs are established for unknown parameters in this section, and ACIs are constructed based on asymptotic theory.

2.1. Maximum Likelihood Estimation. In order to find MLEs of unknown parameters in a concise way, likelihood function (6) can be rewritten in compact expression as

$$
L(\alpha, \beta ; \text { data })=c^{*} \alpha^{d^{*}} \beta^{d^{*}} e^{-\beta w(\alpha)} \prod_{i=1}^{d^{*}} x_{i: m: n}^{\alpha-1},
$$

where $c^{*}=\prod_{j=1}^{d^{*}} \sum_{k=j}^{m}\left(r_{k}+1\right)$, 


$$
\begin{aligned}
& d^{*}= \begin{cases}k, & \text { for case I, } \\
d, & \text { for case II, }, \\
m, & \text { for case III, }\end{cases} \\
& w(\alpha)= \begin{cases}\sum_{i=1}^{d^{*}}\left(1+r_{i}\right) x_{i: m: n}^{\alpha}, & \text { for case I, } \\
\sum_{i=1}^{d^{*}}\left(1+r_{i}\right) x_{i: m: n}^{\alpha}+r_{d+1}^{*} T^{\alpha}, & \text { for case II, } \\
\sum_{i=1}^{d^{*}}\left(1+r_{i}\right) x_{i: m: n}^{\alpha}, & \text { for case III. }\end{cases}
\end{aligned}
$$

From (7), the associated log-likelihood function, namely, $\ell(\alpha, \beta)$, is given by

$$
\ell(\alpha, \beta)=\ln c^{*}+d^{*} \ln \alpha+d^{*} \ln \beta+(\alpha-1) \sum_{i=1}^{d^{*}} \ln x_{i: m: n}-\beta w(\alpha) .
$$

Theorem 1. Suppose that observation (2) comes from Weibull population (5); the MLE of $\beta$ given $\alpha$ can be written as $\widetilde{\beta}=\left(d^{*} / w(\alpha)\right)$.

Proof. See Appendix A.

Furthermore, substituting $\widetilde{\beta}=\tilde{\beta}(\alpha)$ into (9), one has a profile $\log$-likelihood function of $\alpha$, namely, $\ell(\alpha)$, as

$$
\ell(\alpha)=\ln c^{*}+d^{*} \ln \alpha+(\alpha-1) \sum_{i=1}^{d^{*}} \ln x_{i: m: n}+d^{*} \ln \left[\frac{d^{*}}{w(\alpha)}\right]-d^{*} .
$$

The following result establishes the MLE for parameter $\alpha$.

Theorem 2. Suppose that observation (2) comes from Weibull population (5); the MLE of a obtained from (10) uniquely exists which can be derived from the following equation:

$$
\frac{1}{\alpha}+\frac{1}{d^{*}} \sum_{i=1}^{d^{*}} \ln x_{i: m: n}-\frac{w^{\prime}(\alpha)}{w(\alpha)}=0
$$

where

$$
w^{\prime}(\alpha)= \begin{cases}\sum_{i=1}^{k}\left(1+r_{i}\right) x_{i: m: n}^{\alpha} \ln x_{i: m: n}, & \text { for case I, } \\ \sum_{i=1}^{d}\left(1+r_{i}\right) x_{i: m: n}^{\alpha} \ln x_{i: m: n}+r_{d+1}^{*} T^{\alpha} \ln T, & \text { for case II, } \\ \sum_{i=1}^{m}\left(1+r_{i}\right) x_{i: m: n}^{\alpha} \ln x_{i: m: n}, & \text { for case III. }\end{cases}
$$

Proof. See Appendix B.
Denote the MLE of parameter $\alpha$ obtained from (11) in Theorem 2 as $\widehat{\alpha}$; the MLEs of $\beta$, namely, $\widehat{\beta}$, can be further obtained from Theorem 1 as $\widehat{\beta}=\left(d^{*} / w(\widehat{\alpha})\right)$. It is noted that the MLE of $\alpha$ cannot be expressed as closed from (11), and the numerical procedure such as fixed-point iterative algorithm can be used to find the associated estimate.

\subsection{Approximation Confidence Interval Estimation.} Denote $\theta=\left(\theta_{1}, \theta_{2}\right)^{\prime}$ with $\theta_{1}=\alpha, \theta_{2}=\beta$; it is known under mild regularity that the asymptotic distribution of the MLE $\hat{\theta}$ is $\widehat{\theta}-\theta \longrightarrow N\left(0, \mathrm{I}^{-1}(\theta)\right)$, where ' $\longrightarrow$ ' means "distributed as," and $\mathrm{I}^{-1}(\theta)$ is the inverse of the observed information matrix given by

$$
I^{-1}(\widehat{\theta})=\left.\left(-\frac{\partial^{2} \ell(\alpha, \beta)}{\partial \theta_{j} \partial \theta_{k}}\right)_{2 \times 2}^{-1}\right|_{\theta=\widehat{\theta}}=\left(\begin{array}{cc}
\operatorname{Var}(\widehat{\alpha}) & \operatorname{Cov}(\widehat{\alpha}, \widehat{\beta}) \\
\operatorname{Cov}(\widehat{\alpha}, \widehat{\beta}) & \operatorname{Var}(\widehat{\beta})
\end{array}\right),
$$

where the second derivatives of $\ell(\alpha, \beta)$ can be obtained directly which are omitted here for saving space. Therefore, for arbitrary $0<\gamma<1$, the $100(1-\gamma) \%$ ACIs of $\theta_{j}, j=1,2$, is given by

$$
\left(\widehat{\theta}_{j}-z_{\gamma / 2} \sqrt{\operatorname{Var}\left(\hat{\theta}_{j}\right)}, \hat{\theta}_{j}+z_{\gamma / 2} \sqrt{\operatorname{Var}\left(\hat{\theta}_{j}\right)}\right), \quad j=1,2,
$$

where $z_{\gamma}$ is the upper $\gamma$-th quantile of the standard normal distribution.

Sometimes, the ACIs obtained by the previous procedure may have negative lower bounds. In order to overcome this drawback, the logarithmic transformation and delta methods can be used to obtain the asymptotic normality distribution of $\ln \widehat{\theta}_{j}$ as

$$
\frac{\ln \widehat{\theta}_{j}-\ln \theta_{j}}{\operatorname{Var}\left(\ln \widehat{\theta}_{j}\right)} \longrightarrow N(0,1), \quad j=1,2 .
$$

Therefore, a $100(1-\gamma) \%$ ACI of $\theta_{j}, j=1,2$, derived in this manner is provided by

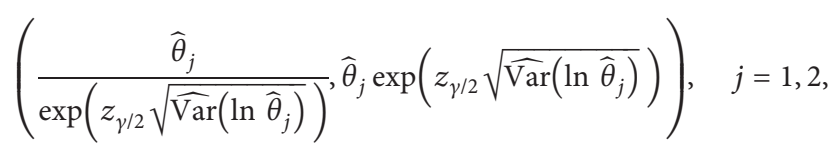

where $\widehat{\operatorname{Var}}\left(\ln \widehat{\theta}_{j}\right)=\widehat{\operatorname{Var}}\left(\widehat{\theta}_{j}\right) / \widehat{\theta}_{j}, j=1,2$.

\section{Bayesian Inference}

As a powerful and valid alternative to classical estimation, the Bayesian viewpoint has received much attention in statistical inference. The capability of incorporating prior information in analysis makes it very valuable in reliability, lifetime study, and other associated fields where one of the major challenges is the limited availability of data.

3.1. Prior Information. In this section, joint prior for $\alpha$ and $\beta$ is considered as follows: 


$$
\pi(\alpha, \beta) \propto \alpha^{a_{1}-1} e^{-b_{1} \alpha} \beta^{a_{2}-1} e^{-b_{2} \beta}, \quad \alpha>0, \beta>0,
$$

where $a_{1}>0, b_{1}>0, a_{2}>0$, and $b_{2}>0$ are hyperparameters. It is seen that the priors of parameters $\alpha$ and $\beta$ are independently gamma-distributed respectively, and the hyperparameters are chosen to reflect prior knowledge. The information abstracted by the priors may be either objective or subjective based on failure data from a history knowledge, personal experience, and belief among others. Momentsmatching and empirical Bayesian methods may be used to fit the prior densities which are not reported here. When $a_{j}=b_{j}=0, j=1,2$, these priors reduce to noninformative and are also nonproper priors. Furthermore, the associated joint posterior density function of $\alpha$ and $\beta$, namely, $\pi(\alpha, \beta \mid$ data $)$, can be expressed as

$$
\pi(\alpha, \beta \mid \text { data })=\frac{\pi(\alpha, \beta) L(\alpha, \beta ; \text { data })}{\int_{0}^{\infty} \int_{0}^{\infty} \pi(\alpha, \beta) L(\alpha, \beta ; \text { data }) \mathrm{d} \alpha \mathrm{d} \beta} .
$$

Therefore, under squared error loss, the Bayes estimator of any function of $\alpha$ and $\beta$, say $\eta(\alpha, \beta)$, is given by

$$
\widehat{\eta}(\alpha, \beta)=\frac{\int_{0}^{\infty} \int_{0}^{\infty} \eta(\alpha, \beta) \pi(\alpha, \beta) L(\alpha, \beta ; \text { data }) \mathrm{d} \alpha \mathrm{d} \beta}{\int_{0}^{\infty} \int_{0}^{\infty} \pi(\alpha, \beta) L(\alpha, \beta ; \text { data }) \mathrm{d} \alpha \mathrm{d} \beta} .
$$

Clearly, the corresponding Bayes estimator of $\eta(\alpha, \beta)$ does not exist in closed form; thus, a Markov Chain Monte Carlo (MCMC) sampling method is used in the following section to compute the associated estimate.

3.2. Posterior Analysis and MCMC Sampling. From (7) and (17) and ignoring the additive constant, joint posterior PDF (18) of $\alpha$ and $\beta$ can be rewritten as

$$
\pi(\alpha, \beta \mid \text { data })=\pi(\alpha \mid \text { data }) \pi(\beta \mid \alpha, \text { data }),
$$

with

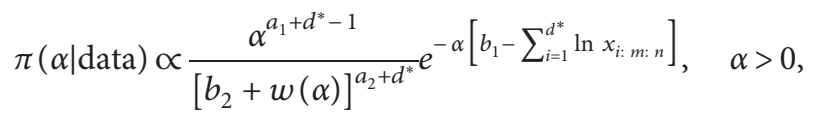

$\pi(\beta \mid \alpha$, data $)=\frac{\left[b_{2}+w(\alpha)\right]^{a_{2}+d^{*}}}{\Gamma\left(a_{2}+d^{*}\right)} \beta^{a_{2}+d^{*}-1} e^{-\beta\left[b_{2}+w(\alpha)\right]}, \quad \beta>0$.

One can observe that for a given $\alpha$, the posterior density function of $\beta$ is gamma-distributed. In addition, by direct computation, one has the following result for $\pi$ ( $\alpha \mid$ data $)$ of which the proof is omitted for concision.

Lemma 1. The posterior marginal PDF $\pi(\alpha \mid$ data $)$ in (21) is log-concave.

In order to generate random samples from the posterior marginal PDF $\pi(\alpha \mid$ data), the Metropolis-Hastings (M-H) algorithm with the gamma proposal distribution is used in our study (see, e.g., Gelman et al. [16]) for the log-concave posterior density. Of course, some other sampling algorithms like the approach for computing the log-concave density (e.g., Devroye [17]) and the adaptive rejection sampling approach (e.g., Gilks and wild [18]) could also be used to generate the sample $\alpha$ from the density $\pi(\alpha \mid$ data) as well. Therefore, the corresponding Bayes estimates as well as the HPD credible intervals can be constructed, and an algorithm is proposed to generate samples and to compute Bayes estimates (Algorithm 1).

\section{Numerical Illustration}

4.1. Simulation Studies. In this section, extensive simulations are carried out for comparing different proposed methods and the performance of the point estimates (i.e., MLEs and Bayes) is evaluated in terms of mean squared error (MSE) and average bias $(\mathrm{AB})$, whereas the confidence interval estimates (i.e., ACIs and HPDs) are compared by criteria coverage probability (CP) and average width (AW), respectively.

The evaluaton criteria quantities for point and interval estimates were examined under different choices of $\beta_{1}, \beta_{2}, \alpha, m, n, \mathrm{CS}, r_{1}, r_{2}, \ldots, r_{m}$, and $T$ values, and the Bayes sampling algorithm was repeated 10,000 times following a burn-in phase of 5,000 iterations. For generating the generalized progressively hybrid censored Weibull samples, it is conducted as the following procedure. Using the algorithm proposed by Balakrishnan and Aggarwala ([19], p. 32), progressively Type-II censored data is generated from Weibull population with parameters $\alpha$ and $\beta$. Then, the generalized progressively hybrid censored sample can be obtained by comparing prefixed $T$ and $X_{k: m: n}$ with those of the generated progressively Type-II censored data.

In the simulation procedure, following CSs are considered:

$$
\begin{aligned}
& \text { CS- } 1: n=18, m=15, r_{1}=\cdots=r_{14}=0, \text { and } r_{15}=3 \\
& \text { CS-2: } n=25, m=15, r_{1}=\cdots=r_{14}=0 \text {, and } r_{15}=10 \\
& \text { CS-3: } n=25, m=20, r_{1}=\cdots=r_{19}=0 \text {, and } r_{20}=5 \\
& \text { CS-4: } n=50, m=40, r_{1}=\cdots=r_{39}=0 \text {, and } r_{40}=10 \\
& \text { CS-5: } n=50, m=40, r_{2}=\cdots=r_{40}=0 \text {, and } r_{1}=10 \\
& \text { CS-6: } \quad n=50, m=40, r_{2}=\cdots=r_{39}=0, \quad \text { and } \\
& r_{1}=r_{40}=5
\end{aligned}
$$

In the simulation procedure, two sets of parameter values are chosen and the Bayes estimates are computed under noninformative prior (NIP) and informative prior (IP) for the Weibull parameters, respectively, where in NIP, the hyperparameters are taken to be $a_{1}=b_{1}=a_{2}=$ $b_{2}=0$ and the hyperparameters of IP are chosen as $a_{1}=$ $1=b_{1}=1, a_{2}=3$, and $b_{2}=5$ for $(\alpha, \beta)=(1,0.6)$ and $a_{1}=$ $3, b_{1}=2$, and $a_{2}=b_{2}=1$ for $(\alpha, \beta)=(1.5,1)$ satisfying that the prior means are as the same as the original mean. The simulation was repeated 10,000 times, and the significance level for interval estimates was taken to be $\gamma=0.05$.

Before starting the numerous simulations, an important process is to investigate the convergence of Bayesian estimation. For parameters $(\alpha, \beta)=(1,0.6)$, we take the CS-1 
Step 1 Generate $\alpha$ from $\pi(\alpha \mid$ data) in (21) by using the proper sampling approach.

Step 2 For given $\alpha$, generate $\beta$ from $\pi(\beta \mid \alpha$, data) in (22).

Step 3 Repeat above Step 1 and Step $2 N$ times, and $N$ samples of $(\alpha, \beta)$ are generated as $\left(\alpha^{k}, \beta^{k}\right), k=1,2, \ldots, N$.

Step 4 The Bayes estimate of $\eta(\alpha, \beta)$ with respect to squared error loss, can be constructed as

$\hat{\eta}(\alpha, \beta)=\left(1 /\left(N-N_{0}\right)\right) \sum_{k=N_{0}+1}^{N} \hat{\eta}_{k}$ and $\hat{\eta}_{k}=\eta\left(\alpha^{k}, \beta^{k}\right)$,

where $N_{0}$ is burn-in period.

Step 5 To construct the HPD credible interval of $\eta(\alpha, \beta)$, first arrange all estimates $\widehat{\eta}_{k}, k=N_{0}+1, N_{0}+2, \ldots, N$ in an ascend order, as $\widehat{\eta}^{[1]}, \widehat{\eta}^{[2]}, \ldots, \widehat{\eta}^{\left[N-N_{0}\right]}$, then for arbitrary $0<\gamma<1$ the $100(1-\gamma)$ credible interval of $\eta(\alpha, \beta)$ can be obtained as $\left(\hat{\eta}^{[k]}, \hat{\eta}^{\left[k+N-N_{0}-\left[\gamma\left(N-N_{0}\right)+1\right]\right]}\right), k=1,2, \ldots,\left[\left(N-N_{0}\right) \gamma\right]$

where $[y]$ denotes the greatest integer less than or equal to $y$. Therefore, the $100(1-\gamma) \%$ HPD credible interval can be constructed as the $k^{*}$ th one satisfying

$\widehat{\eta}^{\left[k^{*}+N-N_{0}-\left[\gamma\left(N-N_{0}\right)+1\right]\right]}-\widehat{\eta}^{\left[k^{*}\right]}=\min _{k=1}^{\left(N-N_{0}\right) \gamma}\left(\widehat{\eta}^{\left[k+N-N_{0}-\left[\gamma\left(N-N_{0}\right)+1\right]\right]}-\widehat{\eta}^{[k]}\right)$

Algorithm 1: MCMC algorithm for Bayes estimation.

and CS-5 with $k=10$ and 35 under $T=0.7$ as a group of demos. A random sample of GPHC is further generated once under each censoring scheme for use in Bayes manner. For space-saving, here we only discuss the Bayes estimates based on IP. Based on M-H algorithm with gamma proposal, the corresponding iterative trend plots are drawn in Figure 2. In a similar way, for parameter $(\alpha, \beta)=(1.5,1)$, we select the CS- 2 and CS- 6 with $k=13$ and 30 under $T=0.9$ as another set of examples. The associated iterative trend plots are also given in Figure 3. It can be seen from Figures 2 and 3 that the mean value of the posterior sample under different parameters and censoring schemes has become stable after $N>5000$, which means that the sampling procedure has a good convergence. Hence, it is noted that the convergence of the MCMC sampling in Bayesian computations is guaranteed in our work.

Furthermore, based on the previous settings and analysis results, the Monte Carlo simulations are carried out and all the numerical results are summarized in Tables 1 to 4 .

From Tables 1-4, the following can be seen for point estimates:

(i) With increase in effective sample size (i.e., $n$ or $m$ or $k$ or their combinations), ABs and MSEs of both MLEs and Bayes estimates for parameters $\alpha$ and $\beta$ decreases

(ii) For the fixed sample size and CS, ABs and MSEs of MLEs and Bayes estimates decrease as monitor time $T$ increases

(iii) MLEs and Bayes estimates with respect to NIP feature similar ABs and MSEs, whereas the Bayes estimates under IP have better performance than the MLEs and Bayes estimates with respect to NIP in terms of ABs and MSEs

Moreover, one can also observe the following for interval estimates:

(i) AWs (CPs) of ACIs and Bayes intervals decrease (increase) as the effective sample size increases (ii) When inspecting time $T$ increases, the AWs (CPs) of ACIs and Bayes credible intervals decrease (increase) generally

(iii) For the fixed sample and CS, AWs of Bayes credible intervals under NIP are slightly shorter than ACIs and the Bayes credible intervals with respect to IP feature smallest AWs among three interval estimates

(iv) Bayes credible intervals from IP have higher CPs than ACIs and Bayes credible intervals under NIP in most cases, where the latter two intervals perform similarly in terms of CPs

In conclusion, from simulation, one can observe that both classic and Bayes estimates have satisfactory behavior and that the performance of Bayes estimates with respect to IP are preferable than MLEs and Bayes results under NIP where the latter two estimates perform similarly.

4.2. Real-Life Illustration. In this part, a set of lifetime data is provided to show the applications of the Weibull model under censored data. From Lawless [20], the number of million revolutions before failure for 25 ball bearings in a life test is used in this illustration and the details are provided in Table 5 .

Before further proceeding, we investigate whether the Weibull distribution can be employed or not to analyze this data. The MLEs of $\alpha$ and $\beta$ under complete data are 0.8143 and 2.1732 , and the Kolmogorov-Smirnov distance and the corresponding $p$ value (within bracket) are 0.1467 (0.6772), respectively. The results indicate that the Weibull distribution provides a reasonable model for this data. Similarly, using Gini statistic, Lee [21] also concluded that the Weibull model can be fitted to this data set. Furthermore, based on the complete failure data from Table 5, censored scheme $r_{1}=$ $\cdots=r_{19}=0, r_{20}=5$ with $k=16, T=1.2$ and $m=20$ is used in advance for the testing; then, the following GPHCS data is generated as 

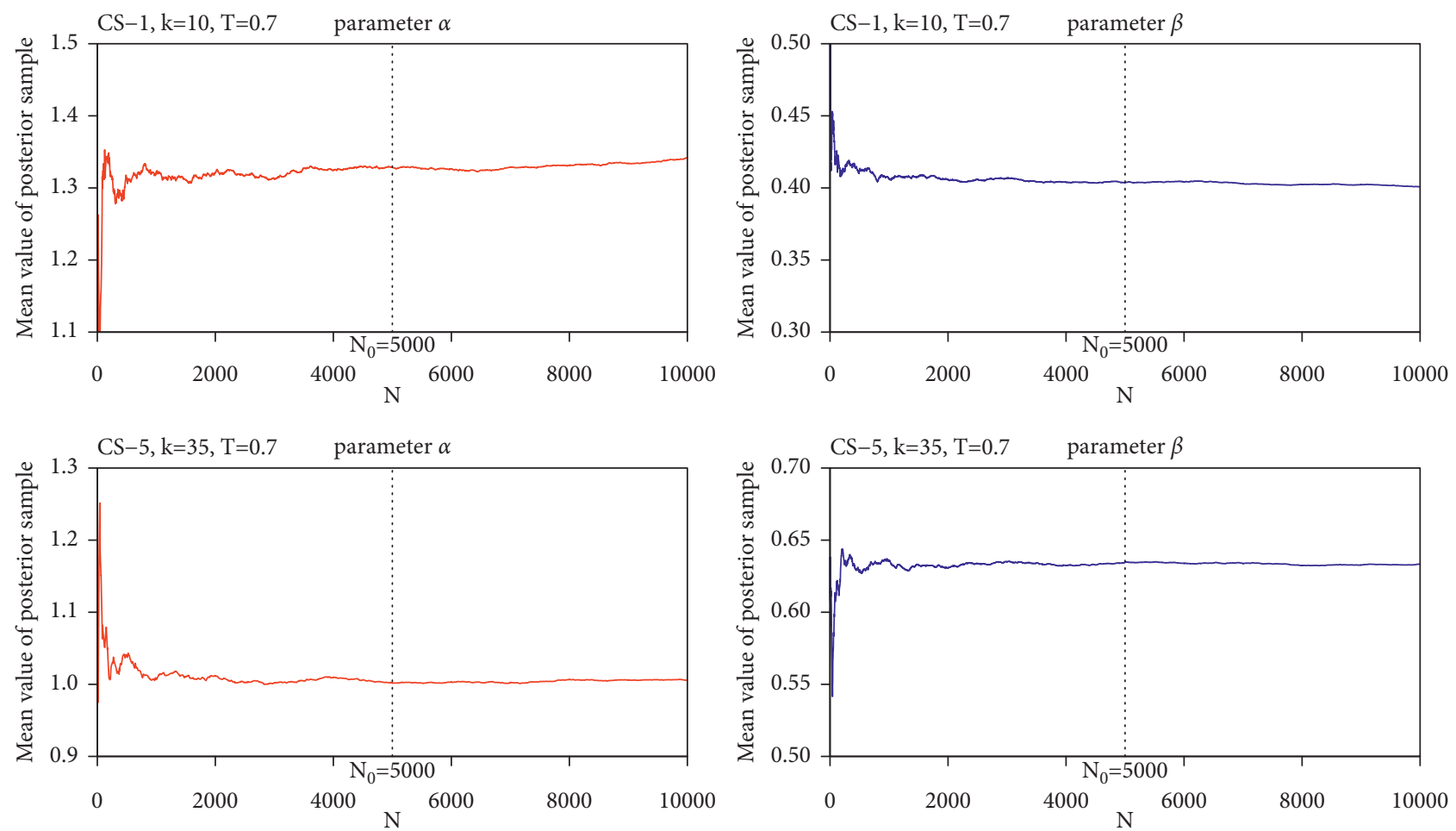

FIgURE 2: The sampling iterative trend plots of parameter $(\alpha, \beta)=(1,0.6)$ under different censoring schemes.
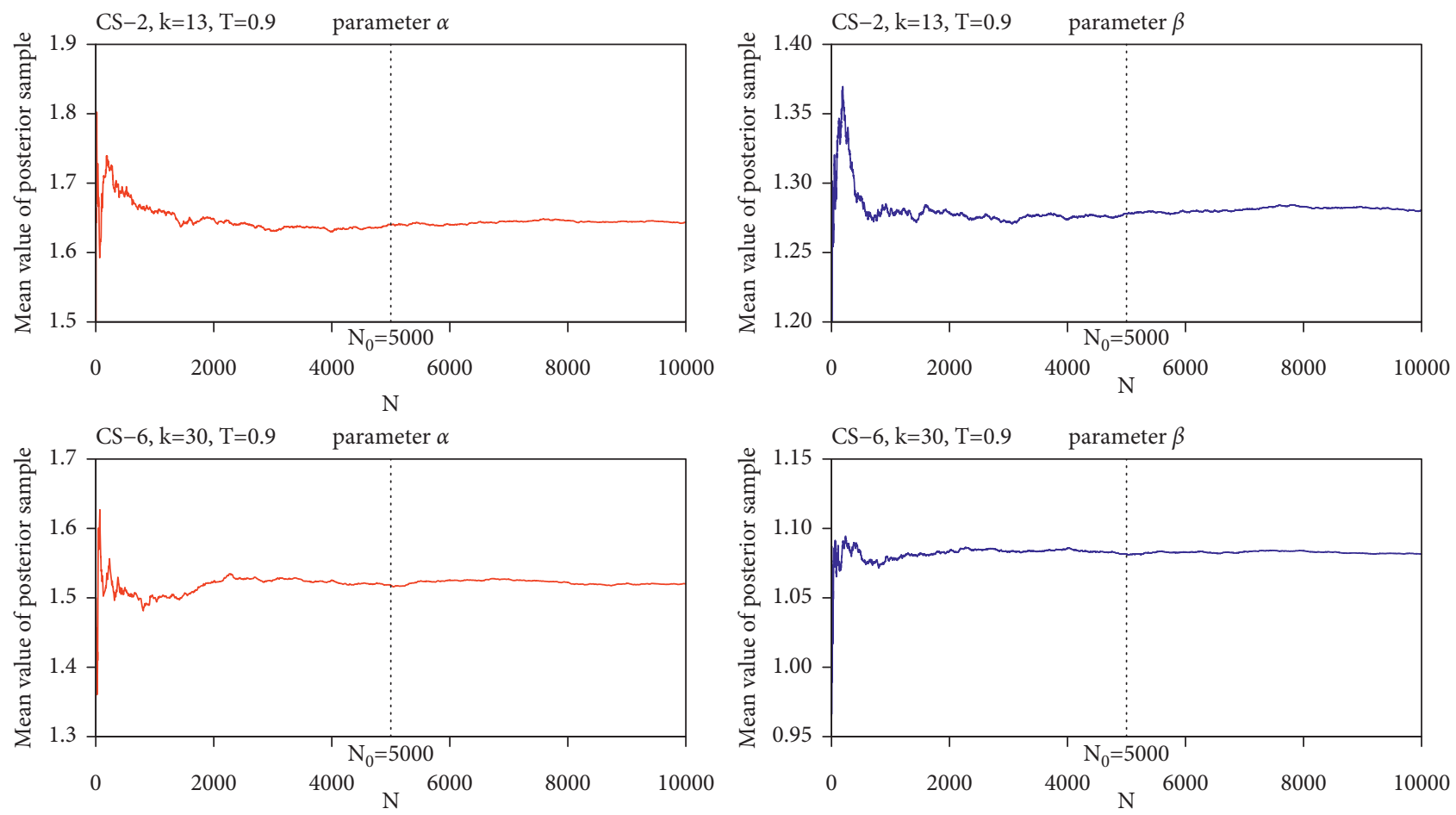

FIgURE 3: The sampling iterative trend plots of parameter $(\alpha, \beta)=(1.5,1)$ under different censoring schemes. 
TABLE 1: ABs and MSEs (within brackets) of the MLEs and Bayes estimates under generalized progressively hybrid censored data with $(\alpha, \beta)=(1,0.6)$.

\begin{tabular}{|c|c|c|c|c|c|c|c|}
\hline \multicolumn{2}{|c|}{$T=0.7$} & \multicolumn{2}{|c|}{ MLE } & \multicolumn{2}{|c|}{ NIP Bayes } & \multicolumn{2}{|c|}{ IP Bayes } \\
\hline CS & $k$ & $\alpha$ & $\beta$ & $\alpha$ & $\beta$ & $\alpha$ & $\beta$ \\
\hline \multirow{2}{*}{1} & 10 & $0.3192[0.2123]$ & $0.2051[0.0861]$ & $0.2858[0.1635]$ & $0.1971[0.0795]$ & $0.2375[0.1044]$ & $0.1313[0.0284]$ \\
\hline & 13 & $0.2497[0.1239]$ & $0.1646[0.0507]$ & $0.2306[0.1027]$ & $0.1581[0.0472]$ & $0.2016[0.0745]$ & $0.1191[0.0239]$ \\
\hline \multirow{2}{*}{2} & 10 & $0.3009[0.1880]$ & $0.1851[0.0669]$ & $0.2707[0.1456]$ & $0.1804[0.0631]$ & $0.2274[0.0955]$ & $0.1216[0.0245]$ \\
\hline & 13 & $0.2603[0.1352]$ & $0.1703[0.0608]$ & $0.2399[0.1115]$ & $0.1658[0.0579]$ & $0.2098[0.0807]$ & $0.1210[0.0249]$ \\
\hline \multirow{2}{*}{3} & 15 & $0.2326[0.1023]$ & $0.1484[0.0428]$ & $0.2167[0.0871]$ & $0.1441[0.0406]$ & $0.1947[0.0679]$ & $0.1125[0.0218]$ \\
\hline & 18 & $0.1992[0.0745]$ & $0.1349[0.0320]$ & $0.1883[0.0654]$ & $0.1309[0.0303]$ & $0.1724[0.0531]$ & $0.1071[0.0193]$ \\
\hline \multirow{2}{*}{4} & 30 & $0.1453[0.0370]$ & $0.0974[0.0162]$ & $0.1407[0.0343]$ & $0.0960[0.0158]$ & $0.1351[0.0313]$ & $0.0858[0.0122]$ \\
\hline & 35 & $0.1325[0.0298]$ & $0.0914[0.0139]$ & $0.1289[0.0279]$ & $0.0900[0.0135]$ & $0.1239[0.0256]$ & $0.0815[0.0109]$ \\
\hline \multirow{2}{*}{5} & 30 & $0.1371[0.0320]$ & $0.0996[0.0165]$ & $0.1333[0.0308]$ & $0.0978[0.0160]$ & $0.1274[0.0272]$ & $0.0869[0.0124]$ \\
\hline & 35 & $0.1205[0.0247]$ & $0.0961[0.0150]$ & $0.1178[0.0234]$ & $0.0945[0.0145]$ & $0.1124[0.0212]$ & $0.0845[0.0115]$ \\
\hline \multirow{2}{*}{6} & 30 & $0.1411[0.0344]$ & $0.0980[0.0163]$ & $0.1369[0.0321]$ & $0.0964[0.0158]$ & $0.1312[0.0292]$ & $0.0861[0.0124]$ \\
\hline & 35 & $0.1247[0.0262]$ & $0.0925[0.0138]$ & $0.1215[0.0247]$ & $0.0911[0.0134]$ & $0.1165[0.0225]$ & $0.0821[0.0108]$ \\
\hline \multicolumn{2}{|c|}{$T=1.2$} & \multicolumn{2}{|c|}{ MLE } & \multicolumn{2}{|c|}{ NIP Bayes } & \multicolumn{2}{|c|}{ IP Bayes } \\
\hline CS & $k$ & $\alpha$ & $\beta$ & $\alpha$ & $\beta$ & $\alpha$ & $\beta$ \\
\hline \multirow{2}{*}{1} & 10 & $0.2780[0.1568]$ & $0.1591[0.0444]$ & $0.2525[0.1237]$ & $0.1535[0.0416]$ & $0.2199[0.0880]$ & $0.1153[0.0223]$ \\
\hline & 13 & $0.2465[0.1193]$ & $0.1613[0.0458]$ & $0.2274[0.0991]$ & $0.1547[0.0426]$ & $0.1986[0.0721]$ & $0.1179[0.0232]$ \\
\hline \multirow{2}{*}{2} & 10 & $0.2420[0.1118]$ & $0.1541[0.0474]$ & $0.2251[0.0935]$ & $0.1519[0.0457]$ & $0.2029[0.0728]$ & $0.1166[0.0234]$ \\
\hline & 13 & $0.2382[0.1095]$ & $0.1463[0.0468]$ & $0.2215[0.0923]$ & $0.1430[0.0448]$ & $0.1988[0.0710]$ & $0.1102[0.0220]$ \\
\hline \multirow{2}{*}{3} & 15 & $0.2213[0.0940]$ & $0.1349[0.0299]$ & $0.2071[0.0805]$ & $0.1311[0.0284]$ & $0.1888[0.0645]$ & $0.1065[0.0182]$ \\
\hline & 18 & $0.1965[0.0719]$ & $0.1316[0.0293]$ & $0.1859[0.0632]$ & $0.1277[0.0277]$ & $0.1700[0.0515]$ & $0.1051[0.0182]$ \\
\hline \multirow{2}{*}{4} & 30 & $0.1449[0.0365]$ & $0.0925[0.0138]$ & $0.1402[0.0338]$ & $0.0912[0.0134]$ & $0.1348[0.0310]$ & $0.0820[0.0108]$ \\
\hline & 35 & $0.1326[0.0306]$ & $0.0908[0.0135]$ & $0.1290[0.0287]$ & $0.0894[0.0132]$ & $0.1241[0.0264]$ & $0.0810[0.0107]$ \\
\hline \multirow{2}{*}{5} & 30 & $0.1369[0.0320]$ & $0.0985[0.0161]$ & $0.1333[0.0301]$ & $0.0968[0.0156]$ & $0.1272[0.0272]$ & $0.0860[0.0121]$ \\
\hline & 35 & $0.1196[0.0241]$ & $0.0959[0.0147]$ & $0.1168[0.0228]$ & $0.0943[0.0143]$ & $0.1113[0.0205]$ & $0.0843[0.0113]$ \\
\hline \multirow{2}{*}{6} & 30 & $0.1431[0.0353]$ & $0.0972[0.0157]$ & $0.1387[0.0328]$ & $0.0956[0.0152]$ & $0.1328[0.0298]$ & $0.0855[0.0120]$ \\
\hline & 35 & $0.1261[0.0273]$ & $0.0933[0.0142]$ & $0.1228[0.0257]$ & $0.0918[0.0138]$ & $0.1177[0.0233]$ & $0.0827[0.0111]$ \\
\hline
\end{tabular}

TABLE 2: AWs and CPs (within brackets) of the ACIs and HPD credible intervals under generalized progressively hybrid censored data with $(\alpha, \beta)=(1,0.6)$.

\begin{tabular}{|c|c|c|c|c|c|c|c|}
\hline \multicolumn{2}{|c|}{$T=0.4$} & \multicolumn{2}{|c|}{$\mathrm{ACI}$} & \multicolumn{2}{|c|}{ NIP HPD } & \multicolumn{2}{|c|}{ IP HPD } \\
\hline $\mathrm{CS}$ & $k$ & $\alpha$ & $\beta$ & $\alpha$ & $\beta$ & $\alpha$ & $\beta$ \\
\hline \multirow{2}{*}{1} & 10 & $1.3707[0.9612]$ & $0.8931[0.9402]$ & $1.2652[0.9545]$ & $0.8489[0.9452]$ & $1.1553[0.9662]$ & $0.6882[0.9754]$ \\
\hline & 13 & $1.0994[0.9554]$ & $0.7405[0.9293]$ & $1.0361[0.9485]$ & $0.7157[0.9365]$ & $0.9688[0.9580]$ & $0.6253[0.9680]$ \\
\hline \multirow{2}{*}{2} & 10 & $1.3494[0.9635]$ & $0.9018[0.9535]$ & $1.2441[0.9548]$ & $0.8535[0.9474]$ & $1.1356[0.9648]$ & $0.6872[0.9697]$ \\
\hline & 13 & $1.1525[0.9551]$ & $0.7577[0.9460]$ & $1.0802[0.9493]$ & $0.7284[0.9422]$ & $1.0134[0.9585]$ & $0.6195[0.9658]$ \\
\hline \multirow{2}{*}{3} & 15 & $1.0397[0.9590]$ & $0.6731[0.9443]$ & $0.9827[0.9521]$ & $0.6520[0.9432]$ & $0.9337[0.9608]$ & $0.5779[0.9674]$ \\
\hline & 18 & $0.8984[0.9513]$ & $0.6162[0.9326]$ & $0.8562[0.9463]$ & $0.6006[0.9375]$ & $0.8191[0.9546]$ & $0.5455[0.9616]$ \\
\hline \multirow{2}{*}{4} & 30 & $0.6868[0.9525]$ & $0.4534[0.9460]$ & $0.6599[0.9451]$ & $0.4452[0.9431]$ & $0.6469[0.9496]$ & $0.4215[0.9563]$ \\
\hline & 35 & $0.6172[0.9522]$ & $0.4287[0.9375]$ & $0.5950[0.9420]$ & $0.4219[0.9406]$ & $0.5832[0.9459]$ & $0.4020[0.9513]$ \\
\hline \multirow{2}{*}{5} & 30 & $0.6409[0.9540]$ & $0.4700[0.9395]$ & $0.6168[0.9446]$ & $0.4609[0.9427]$ & $0.6024[0.9490]$ & $0.4352[0.9548]$ \\
\hline & 35 & $0.5667[0.9520]$ & $0.4583[0.9398]$ & $0.5461[0.9429]$ & $0.4499[0.9412]$ & $0.5330[0.9468]$ & $0.4259[0.9548]$ \\
\hline \multirow{2}{*}{6} & 30 & $0.6674[0.9553]$ & $0.4582[0.9375]$ & $0.6414[0.9482]$ & $0.4497[0.9391]$ & $0.6279[0.9495]$ & $0.4256[0.9518]$ \\
\hline & 35 & $0.5937[0.9531]$ & $0.4404[0.9414]$ & $0.5717[0.9442]$ & $0.4329[0.9443]$ & $0.5599[0.9503]$ & $0.4115[0.9561]$ \\
\hline \multicolumn{2}{|c|}{$T=1.2$} & \multicolumn{2}{|c|}{ ACI } & \multicolumn{2}{|c|}{ NIP HPD } & \multicolumn{2}{|c|}{ IP HPD } \\
\hline $\mathrm{CS}$ & $k$ & $\alpha$ & $\beta$ & $\alpha$ & $\beta$ & $\alpha$ & $\beta$ \\
\hline \multirow{2}{*}{1} & 10 & $1.2697[0.9604]$ & $0.7774[0.9302]$ & $1.1790[0.9549]$ & $0.7463[0.9351]$ & $1.1010[0.9636]$ & 0.6485 [0.9649] \\
\hline & 13 & $1.0967[0.9591]$ & $0.7309[0.9228]$ & $1.0337[0.9512]$ & $0.7069[0.9327]$ & $0.9677[0.9614]$ & $0.6214[0.9641]$ \\
\hline \multirow{2}{*}{2} & 10 & $1.1252[0.9532]$ & $0.7021[0.9553]$ & $1.0540[0.9466]$ & $0.6768[0.9421]$ & $1.0019[0.9561]$ & $0.5960[0.9702]$ \\
\hline & 13 & $1.0929[0.9589]$ & $0.6918[0.9448]$ & $1.0284[0.9505]$ & $0.6684[0.9400]$ & $0.9768[0.9585]$ & $0.5885[0.9650]$ \\
\hline \multirow{2}{*}{3} & 15 & $1.0126[0.9576]$ & $0.6441[0.9399]$ & $0.9583[0.9507]$ & $0.6252[0.9424]$ & $0.9156[0.9584]$ & $0.5646[0.9644]$ \\
\hline & 18 & $0.8972[0.9548]$ & $0.6111[0.9365]$ & $0.8553[0.9506]$ & $0.5957[0.9416]$ & $0.8182[0.9555]$ & $0.5424[0.9640]$ \\
\hline \multirow[b]{2}{*}{4} & 30 & $0.6849[0.9546]$ & $0.4482[0.9492]$ & $0.6584[0.9458]$ & $0.4403[0.9506]$ & $0.6455[0.9517]$ & $0.4180[0.9604]$ \\
\hline & 35 & $0.6176[0.9456]$ & $0.4287[0.9409]$ & $0.5953[0.9354]$ & $0.4221[0.9433]$ & $0.5833[0.9427]$ & $0.4021[0.9556]$ \\
\hline \multirow{2}{*}{5} & 30 & $0.6408[0.9523]$ & $0.4696[0.9427]$ & $0.6167[0.9439]$ & $0.4605[0.9428]$ & $0.6024[0.9491]$ & $0.4348[0.9569]$ \\
\hline & 35 & $0.5657[0.9522]$ & $0.4590[0.9407]$ & $0.5453[0.9440]$ & $0.4506[0.9422]$ & $0.5318[0.9481]$ & $0.4265[0.9565]$ \\
\hline \multirow{2}{*}{6} & 30 & $0.6688[0.953] 0$ & $0.4567[0.9389]$ & $0.6432[0.9459]$ & 0.4483 [0.937 9] & $0.6298[0.9502]$ & $0.4246[0.9515]$ \\
\hline & 35 & $0.5952[0.9492]$ & $0.4403[0.9372]$ & $0.5731[0.9394]$ & $0.4329[0.9380]$ & $0.5613[0.9459]$ & $0.4016[0.9507]$ \\
\hline
\end{tabular}


TABLE 3: ABs and MSEs (within brackets) of the MLEs and Bayes estimates under generalized progressively hybrid censored data with $(\alpha, \beta)=(1.5,1)$.

\begin{tabular}{|c|c|c|c|c|c|c|c|}
\hline \multicolumn{2}{|c|}{$T=0.4$} & \multicolumn{2}{|c|}{ MLE } & \multicolumn{2}{|c|}{ NIP Bayes } & \multicolumn{2}{|c|}{ IP Bayes } \\
\hline $\mathrm{CS}$ & $k$ & $\alpha$ & $\beta$ & $\alpha$ & $\beta$ & $\alpha$ & $\beta$ \\
\hline \multirow{2}{*}{1} & 10 & $0.4963[0.5125]$ & $0.5636[0.6318]$ & $0.4146[0.3353]$ & $0.5289[0.5736]$ & $0.2862[0.1386]$ & $0.2960[0.1709]$ \\
\hline & 13 & $0.3834[0.2861]$ & $0.3070[0.2859]$ & $0.3373[0.2126]$ & $0.2921[0.2605]$ & $0.2677[0.1233]$ & $0.2410[0.1144]$ \\
\hline \multirow{2}{*}{2} & 10 & $0.4875[0.4957]$ & $0.6517[0.7554]$ & $0.4056[0.3202]$ & $0.6023[0.5817]$ & $0.2639[0.1158]$ & $0.3031[0.1673]$ \\
\hline & 13 & $0.3957[0.3114]$ & $0.3900[0.5735]$ & $0.3459[0.2271]$ & $0.3742[0.5268]$ & $0.2608[0.1164]$ & $0.2621[0.1341]$ \\
\hline \multirow{2}{*}{3} & 15 & $0.3472[0.2352]$ & $0.2976[0.2329]$ & $0.3099[0.1806]$ & $0.2857[0.2111]$ & $0.2494[0.1080]$ & $0.2330[0.1056]$ \\
\hline & 18 & $0.2951[0.1623]$ & $0.2295[0.1151]$ & $0.2711[0.1324]$ & $0.2219[0.1067]$ & $0.2319[0.0925]$ & $0.1999[0.0762]$ \\
\hline \multirow{2}{*}{4} & 30 & $0.2195[0.0835]$ & $0.1790[0.0647]$ & $0.2082[0.0737]$ & 0.1757 [0.0619] & $0.1887[0.0593]$ & $0.1636[0.0502]$ \\
\hline & 35 & $0.1978[0.0671]$ & $0.1512[0.0409]$ & $0.1891[0.0604]$ & $0.1487[0.0394]$ & $0.1753[0.0512]$ & $0.1426[0.0354]$ \\
\hline \multirow{2}{*}{5} & 30 & $0.2028[0.0696]$ & $0.1639[0.0488]$ & $0.1938[0.0626]$ & $0.1608[0.0467]$ & $0.1789[0.0526]$ & $0.1536[0.0415]$ \\
\hline & 35 & $0.1814[0.0555]$ & $0.1440[0.0352]$ & $0.1751[0.0509]$ & $0.1415[0.0339]$ & $0.1643[0.0444]$ & $0.1368[0.0315]$ \\
\hline \multirow{2}{*}{6} & 30 & $0.2111[0.0766]$ & $0.1683[0.0540]$ & $0.2009[0.0683]$ & $0.1652[0.0517]$ & $0.1835[0.0560]$ & $0.1562[0.0441]$ \\
\hline & 35 & $0.1904[0.0623]$ & $0.1491[0.0390]$ & $0.1831[0.0566]$ & $0.1465[0.0375]$ & $0.1707[0.0486]$ & $0.1413[0.0343]$ \\
\hline \multicolumn{2}{|c|}{$T=0.9$} & \multicolumn{2}{|c|}{ MLE } & \multicolumn{2}{|c|}{ NIP Bayes } & \multicolumn{2}{|c|}{ IP Bayes } \\
\hline CS & $k$ & $\alpha$ & $\beta$ & $\alpha$ & $\beta$ & $\alpha$ & $\beta$ \\
\hline \multirow{2}{*}{1} & 10 & $0.3884[0.2956]$ & $0.2698[0.1563]$ & $0.3380[0.2099]$ & $0.2591[0.1406]$ & $0.2626[0.1158]$ & $0.2234[0.0921]$ \\
\hline & 13 & $0.3590[0.2474]$ & $0.2753[0.1560]$ & $0.3181[0.1861]$ & $0.2622[0.1406]$ & $0.2570[0.1133]$ & $0.2293[0.0954]$ \\
\hline \multirow{2}{*}{2} & 10 & $0.3517[0.2376]$ & $0.3205[0.2737]$ & $0.3160[0.1825]$ & $0.3114[0.2509]$ & $0.2510[0.1061]$ & $0.2532[0.1177]$ \\
\hline & 13 & $0.3577[0.2420]$ & $0.3154[0.2852]$ & $0.3198[0.1852]$ & $0.3057[0.2606]$ & $0.2544[0.1087]$ & $0.2471[0.1149]$ \\
\hline \multirow{2}{*}{3} & 15 & $0.3125[0.1811]$ & $0.2247[0.0961]$ & $0.2843[0.1438]$ & $0.2171[0.0894]$ & $0.2381[0.0960]$ & $0.1959[0.0689]$ \\
\hline & 18 & $0.2881[0.1513]$ & $0.2247[0.0976]$ & $0.2639[0.1235]$ & $0.2170[0.0904]$ & $0.2270[0.0878]$ & $0.1983[0.0706]$ \\
\hline \multirow{2}{*}{4} & 30 & $0.2099[0.0762]$ & $0.1504[0.0380]$ & $0.2002[0.0680]$ & $0.1479[0.0367]$ & $0.1836[0.0563]$ & $0.1413[0.0331]$ \\
\hline & 35 & $0.1962[0.0654]$ & $0.1472[0.0374]$ & $0.1877[0.0589]$ & $0.1446[0.0360]$ & $0.1741[0.0501]$ & $0.1390[0.0328]$ \\
\hline \multirow{2}{*}{5} & 30 & $0.2044[0.0724]$ & $0.1624[0.0474]$ & $0.1947[0.0648]$ & $0.1593[0.0454]$ & $0.1799[0.0545]$ & $0.1522[0.0406]$ \\
\hline & 35 & $0.1795[0.0547]$ & $0.1468[0.0375]$ & $0.1729[0.0501]$ & $0.1442[0.0361]$ & $0.1624[0.0438]$ & $0.1392[0.0334]$ \\
\hline \multirow[b]{2}{*}{6} & 30 & $0.2097[0.0758]$ & $0.1631[0.0452]$ & $0.2000[0.0677]$ & $0.1601[0.0433]$ & $0.1835[0.0561]$ & $0.1526[0.0388]$ \\
\hline & 35 & $0.1899[0.0608]$ & $0.1466[0.0377]$ & $0.1825[0.0553]$ & $0.1440[0.0362]$ & $0.1704[0.0476]$ & $0.1389[0.0332]$ \\
\hline
\end{tabular}

TABLE 4: AWs and CPs (within brackets) of the ACIs and HPD credible intervals under generalized progressively hybrid censored data with $(\beta, \alpha)=(1.5,1)$.

\begin{tabular}{|c|c|c|c|c|c|c|c|}
\hline \multicolumn{2}{|c|}{$T=0.4$} & \multicolumn{2}{|c|}{ ACI } & \multicolumn{2}{|c|}{ NIP HPD } & \multicolumn{2}{|c|}{ IP HPD } \\
\hline CS & $k$ & $\alpha$ & $\beta$ & $\alpha$ & $\beta$ & $\alpha$ & $\beta$ \\
\hline \multirow{2}{*}{1} & 10 & $2.0759[0.9589]$ & $2.5932[0.9610]$ & $1.8700[0.9574]$ & $2.1754[0.9605]$ & $1.5519[0.9789]$ & $1.4488[0.9664]$ \\
\hline & 13 & $1.6552[0.9533]$ & $1.3475[0.9553]$ & $1.5365[0.9502]$ & $1.2761[0.9512]$ & $1.3706[0.9690]$ & $1.1503[0.9616]$ \\
\hline \multirow{2}{*}{2} & 10 & $2.1199[0.9640]$ & $3.2946[0.9681]$ & $1.8981[0.9606]$ & $2.7963[0.9663]$ & $1.5210[0.9816]$ & $1.6133[0.9719]$ \\
\hline & 13 & $1.7412[0.9547]$ & $1.7686[0.9689]$ & $1.6053[0.9525]$ & $1.6274[0.9662]$ & $1.3928[0.9720]$ & $1.2977[0.9706]$ \\
\hline \multirow{2}{*}{3} & 15 & $1.5554[0.9569]$ & $1.3430[0.9642]$ & $1.4512[0.9540]$ & $1.2684[0.9571]$ & $1.3031[0.9705]$ & $1.1285[0.9647]$ \\
\hline & 18 & $1.3396[0.9536]$ & $1.0496[0.9590]$ & $1.2662[0.9481]$ & $1.0123[0.9494]$ & $1.1727[0.9644]$ & $0.9611[0.9563]$ \\
\hline \multirow{2}{*}{4} & 30 & $1.0312[0.9544]$ & $0.8283[0.9561]$ & $0.9874[0.9480]$ & $0.8065[0.9475]$ & $0.9402[0.9576]$ & $0.7776[0.9514]$ \\
\hline & 35 & $0.9263[0.9527]$ & $0.7080[0.9497]$ & $0.8905[0.9475]$ & $0.6937[0.9464]$ & $0.8574[0.9527]$ & $0.6803[0.9514]$ \\
\hline \multirow{2}{*}{5} & 30 & $0.9595[0.9526]$ & $0.7631[0.9510]$ & $0.9208[0.9477]$ & $0.7462[0.9493]$ & $0.8844[0.9548]$ & $0.7303[0.9527]$ \\
\hline & 35 & $0.8477[0.9498]$ & $0.6916[0.9469]$ & $0.8167[0.9441]$ & $0.6793[0.9462]$ & $0.7906[0.9505]$ & $0.6684[0.9516]$ \\
\hline \multirow[b]{2}{*}{6} & 30 & $0.9987[0.9554]$ & $0.7896[0.9589]$ & $0.9564[0.9504]$ & $0.7708[0.9527]$ & $0.9155[0.9571]$ & $0.7497[0.9565]$ \\
\hline & 35 & $0.8916[0.9506]$ & $0.6958[0.9489]$ & $0.8574[0.9439]$ & $0.6829[0.9466]$ & $0.8287[0.9518]$ & $0.6711\left[\begin{array}{ll}0.9503 \\
\end{array}\right.$ \\
\hline \multicolumn{2}{|c|}{$T=0.9$} & \multicolumn{2}{|c|}{$\mathrm{ACI}$} & \multicolumn{2}{|c|}{ NIP HPD } & \multicolumn{2}{|c|}{ IP HPD } \\
\hline $\mathrm{CS}$ & $k$ & $\alpha$ & $\beta$ & $\alpha$ & $\beta$ & $\alpha$ & $\beta$ \\
\hline \multirow{2}{*}{1} & 10 & $2.0759[0.9589]$ & $2.5932[0.9610]$ & $1.8700[0.9574]$ & $2.1754[0.9605]$ & $1.5519[0.9789]$ & $1.4488[0.9664]$ \\
\hline & 13 & $1.6552[0.9533]$ & $1.3475[0.9553]$ & $1.5365[0.9502]$ & $1.2761[0.9512]$ & $1.3706[0.9690]$ & $1.1503[0.9616]$ \\
\hline \multirow{2}{*}{2} & 10 & $2.1199[0.9640]$ & $3.2946[0.9681]$ & $1.8981[0.9606]$ & $2.7963[0.9663]$ & $1.5210[0.9816]$ & $1.6133[0.9719]$ \\
\hline & 13 & $1.7412[0.9547]$ & $1.7686[0.9689]$ & $1.6053[0.9525]$ & $1.6274[0.9662]$ & $1.3928[0.9720]$ & $1.2977[0.9706]$ \\
\hline \multirow{2}{*}{3} & 15 & $1.5554[0.9569]$ & $1.3430[0.9642]$ & $1.4512[0.9540]$ & $1.2684[0.9571]$ & $1.3031[0.9705]$ & $1.1285[0.9647]$ \\
\hline & 18 & $1.3396[0.9536]$ & $1.0496[0.9590]$ & $1.2662[0.9481]$ & $1.0123[0.9494]$ & $1.1727[0.9644]$ & $0.9611[0.9563]$ \\
\hline \multirow[b]{2}{*}{4} & 30 & $1.0312[0.9544]$ & $0.8283[0.9561]$ & $0.9874[0.9480]$ & $0.8065[0.9475]$ & $0.9402[0.9576]$ & $0.7776[0.9514]$ \\
\hline & 35 & $0.9263[0.9527]$ & $0.7080[0.9497]$ & $0.8905[0.9475]$ & $0.6937[0.9464]$ & $0.8574[0.9527]$ & $0.6803[0.9514]$ \\
\hline \multirow{2}{*}{5} & 30 & $0.9595[0.9526]$ & $0.7631[0.9510]$ & $0.9208[0.9477]$ & $0.7462[0.9493]$ & $0.8844[0.9548]$ & $0.7303[0.9527]$ \\
\hline & 35 & $0.8477[0.9498]$ & $0.6916[0.9469]$ & $0.8167[0.9441]$ & $0.6793[0.9462]$ & $0.7906[0.9505]$ & $0.6684[0.9516]$ \\
\hline \multirow[b]{2}{*}{6} & 30 & $0.9987[0.9554]$ & $0.7896[0.9589]$ & $0.9564[0.9504]$ & $0.7708[0.9527]$ & $0.9155[0.9571]$ & $0.7497[0.9565]$ \\
\hline & 35 & $0.8916[0.9506]$ & $0.6958[0.9489]$ & $0.8574[0.9439]$ & $0.6829[0.9466]$ & $0.8287[0.9518]$ & $0.6711[0.9503]$ \\
\hline
\end{tabular}


TABLE 5: The number of million revolutions before failure for ball bearings.

\begin{tabular}{|c|c|c|c|c|c|c|c|c|c|}
\hline 0.1788 & 0.2892 & 0.3300 & 0.4152 & 0.4212 & 0.4560 & 0.4848 & 0.5184 & 0.5196 & 0.5412 \\
\hline 0.5556 & 0.6780 & 0.6780 & 0.6780 & 0.6864 & 0.6864 & 0.6888 & 0.8412 & 0.9312 & 0.9864 \\
\hline 1.0512 & 1.0584 & 1.2792 & 1.2804 & 1.7340 & & & & & \\
\hline
\end{tabular}

TABle 6: Point and interval estimates of $\alpha$ and $\beta$ under real application data.

\begin{tabular}{lcr}
\hline & $\alpha$ & $\beta$ \\
\hline MLE & $2.4233[0.4546]$ & $1.7772[0.4310]$ \\
Bayes & $2.3723[0.4401]$ & $1.7519[0.4282]$ \\
ACI & $(1.5187,3.2897)[1.7819]$ & $(0.9324,2.6221)[1.6897]$ \\
HPD & $(1.5126,3.2177)[1.7051]$ & $(0.9417,2.5581)[1.6164]$ \\
\hline
\end{tabular}

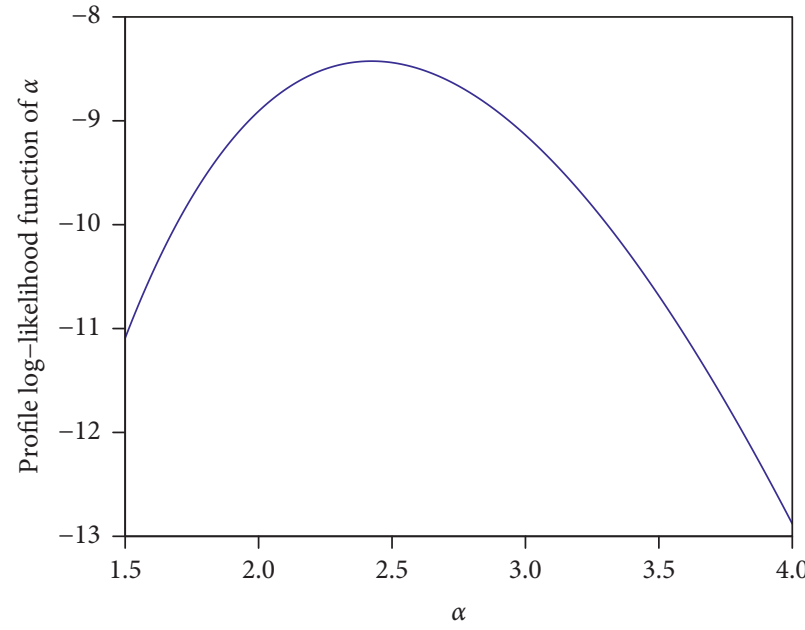

(a)

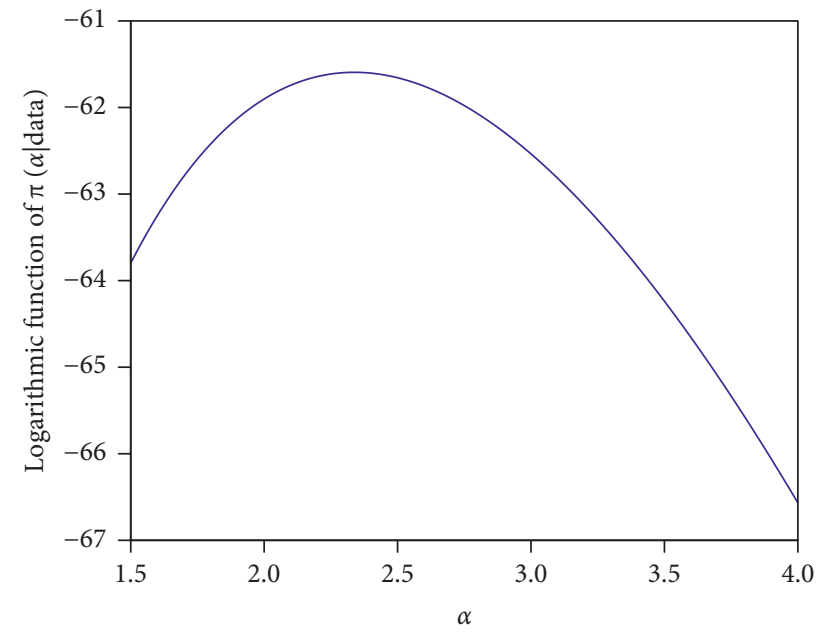

(b)

FIGURE 4: The profile log-likelihood (a) and the posterior marginal density function (b) of $\alpha$ under ball-bearings failure data.
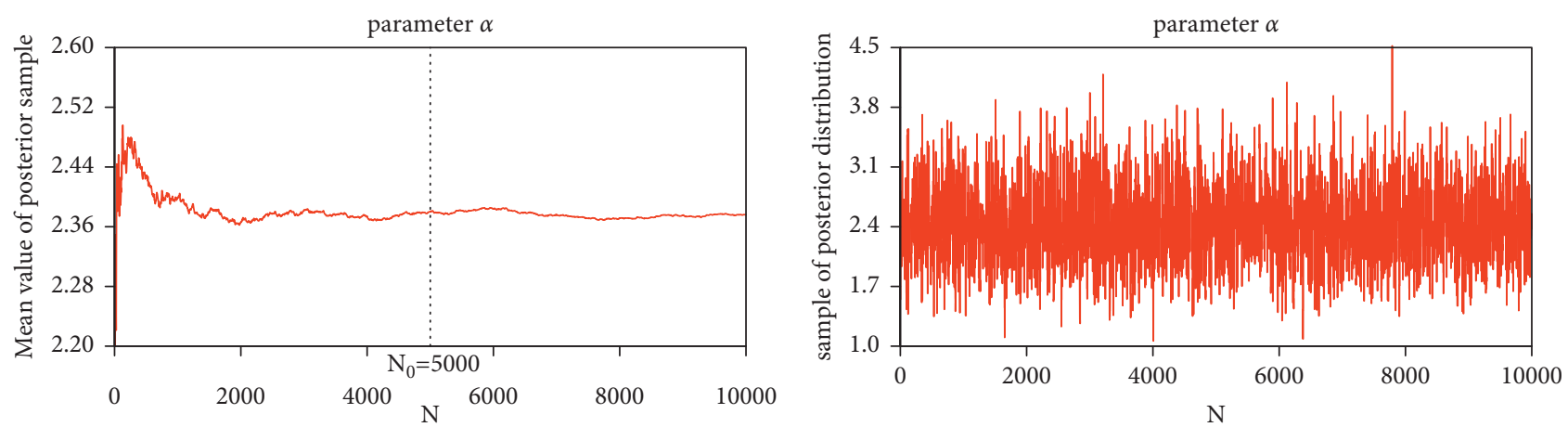

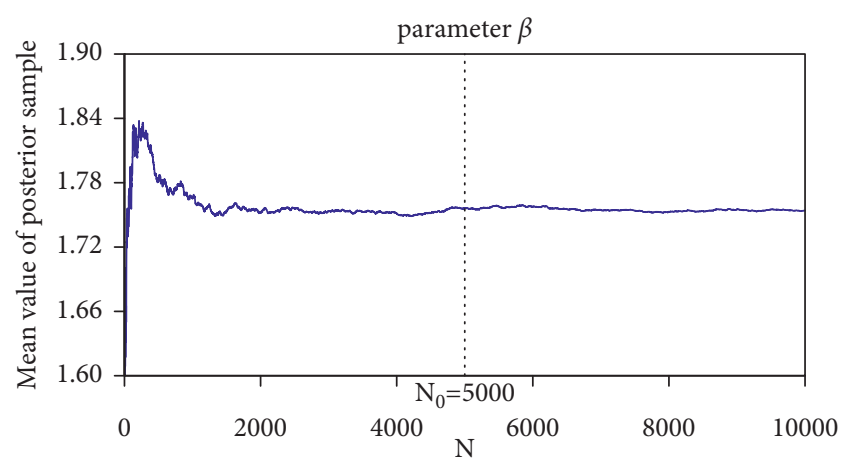

(a)

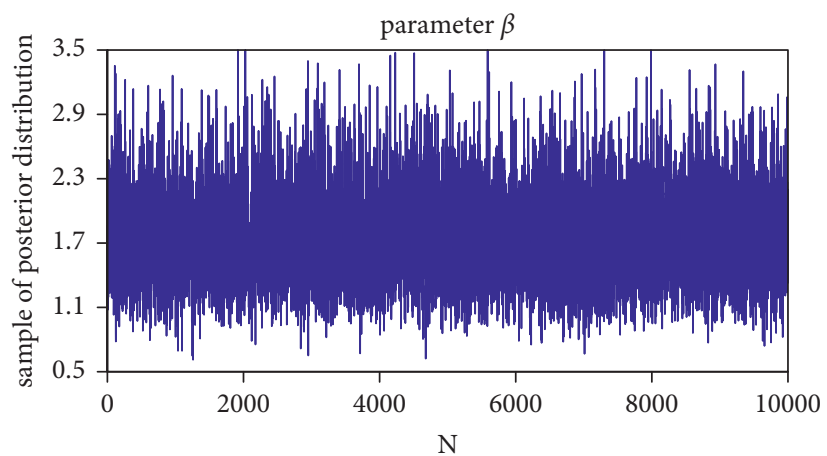

(b)

Figure 5: The iterative trend plots (a) and the trace plots (b) of sampling algorithm for parameter $\alpha$ and $\beta$ under ball-bearings failure data. 


$\begin{array}{llllllllll}0.1788 & 0.2892 & 0.3300 & 0.4152 & 0.4212 & 0.4560 & 0.4848 & 0.5184 & 0.5196 & 0.5412 \\ 0.5556 & 0.6780 & 0.6780 & 0.6780 & 0.6864 & 0.6864 & 0.6888 & 0.8412 & 0.9312 & 0.9864\end{array}$.

It is noted that the obtained GPHCS data is the common Type-II censored sample which is the most occurred failure observation in practice. Therefore, the corresponding point and interval estimates are calculated, and the results are listed in Table 6 with a significance level $\gamma=0.05$. To compare the performance of different estimates, the estimated standard errors (ESEs) for point estimates and the interval lengths for interval estimates are shown in squared brackets, respectively. Since there is no information about the unknown parameters, NIP with $a_{1}=b_{1}=a_{2}=b_{2}=0$ is used in this illustrative example. Based on the results in Table 6, one could observe that although the MLEs and Bayes estimates of the parameters $\alpha$ and $\beta$ are close to each other, the ESE of Bayes results is smaller than that of MLE. In addition, the Bayes credible intervals under NIP also have better performance than ACIs in terms of interval length. Besides, to illustrate Theorem 2 and Lemma 1, the curves of the profile log-likelihood function (10) and the logarithmic function of posterior marginal density (21) with respect to NIP are plotted in Figure 4 under the censored failure data. It is observed that the profile log-likelihood function of $\alpha$ has a unique maximum point and the posterior marginal density function $\pi(\alpha \mid \mathrm{data})$ is log-concave. Finally, the trace plots and iterative trend plots of Bayes sampling algorithm are given in Figure 5. One could also note that the MCMC sampling in Bayesian computations converges in the real-life illustration.

\section{Concluding Remarks}

In this paper, classical and Bayesian inferences are considered for generalized progressive hybrid censored data. When the latent failure times are Weibull distributed, point and interval estimations are discussed for the unknown parameters, respectively. The existence and uniqueness of MLEs are established, and Bayes estimates are obtained by using the Monte Carlo sampling method. Numerical illustration indicates that classical and Bayes estimates have similar performance when noninformative prior is adopted and that the Bayes estimators with respect to informative prior perform better than MLEs and Bayes estimators under noninformative prior. Furthermore, as a future work, optimal design and sampling plan of generalized progressive hybrid censoring under Weibull population seem also of interest which will be studied in the future.

\section{Appendix}

\section{A. The Proof of Theorem 1}

Taking the derivatives of $\ell(\beta, \alpha)$ with respect to $\beta$ and equating them to zero, one directly has $\beta=\left(d^{*} / w(\alpha)\right)$. Moreover, since $\left.\left(\partial^{2} \ell(\beta, \alpha) / \partial \beta^{2}\right)\right|_{\beta=\tilde{\beta}}=-\left(d^{*} / \widetilde{\beta}^{2}\right)<0$, then $\tilde{\beta}$ is the local maximum of $\ell(\beta, \alpha)$ for the given $\alpha$. Since there is no singular point of $\ell(\beta, \alpha)$ and it has a single critical point, $\widetilde{\beta}$ is the absolute maximum of $\ell(\beta, \alpha)$. Therefore, the assertion is proved.

\section{B. The Proof of Theorem 2}

Taking derivative for (10) and setting it to zero, one has likelihood equation (11). Next, it is shown that equation (11) has a unique root with respect to $\alpha$.

Denote

$$
\begin{aligned}
& G_{1}(\alpha)=\frac{1}{\alpha}, \\
& G_{2}(\alpha)=\frac{w^{\prime}(\alpha)}{w(\alpha)}-\frac{1}{d^{*}} \sum_{i=1}^{d^{*}} \ln x_{i: m: n} .
\end{aligned}
$$

It is seen that $G_{1}(\alpha)$ decreases in $\alpha$ and $G_{1}(0)=+\infty$ and $G_{1}(\infty)=0$. Meanwhile, for $G_{2}(\alpha)$, using Cauchy-Schwartz inequality, one can observe under cases I, II, and III, respectively, that

$$
\frac{\mathrm{d} G_{2}(\alpha)}{\mathrm{d} \alpha}=\frac{w(\alpha) w^{\prime \prime}(\alpha)-\left[w^{\prime}(\alpha)\right]^{2}}{[w(\alpha)]^{2}} \geq 0,
$$

with

$$
w^{\prime \prime}(\alpha)= \begin{cases}\sum_{i=1}^{k}\left(1+r_{i}\right) x_{i: m: n}^{\alpha} \ln ^{2} x_{i: m: n}, & \text { for case I, } \\ \sum_{i=1}^{d}\left(1+r_{i}\right) x_{i: m: n}^{\alpha} \ln ^{2} x_{i: m: n}+r_{d+1}^{*} T^{\alpha} \ln ^{2} T, & \text { for case II, } \\ \sum_{i=1}^{m}\left(1+r_{i}\right) x_{i: m: n}^{\alpha} \ln ^{2} x_{i: m: n}, & \text { for case III, }\end{cases}
$$

which implies that $G_{2}(\alpha)$ increases in $\alpha$. Moreover, since 


$$
\begin{aligned}
& \lim _{\alpha \longrightarrow 0} G_{2}(\alpha)= \begin{cases}\frac{1}{d^{*}} \sum_{i=1}^{d^{*}} \ln x_{i: m: n}-\frac{\sum_{i=1}^{k}\left(1+r_{i}\right) \ln x_{i: m: n}}{\sum_{i=1}^{k}\left(1+r_{i}\right)}, & \text { for case I, } \\
\frac{1}{d^{*}} \sum_{i=1}^{d^{*}} \ln x_{i: m: n}-\frac{\sum_{i=1}^{d}\left(1+r_{i}\right) \ln x_{i: m: n}+r_{d+1}^{*} \ln T}{\sum_{i=1}^{d}\left(1+r_{i}\right)+r_{d+1}^{*}}, & \text { for case II, } \\
\frac{1}{d^{*}} \sum_{i=1}^{d^{*}} \ln x_{i: m: n}-\frac{\sum_{i=1}^{m}\left(1+r_{i}\right) \ln x_{i: m: n}}{\sum_{i=1}^{m}\left(1+r_{i}\right)}, & \text { for case III, }\end{cases}
\end{aligned}
$$

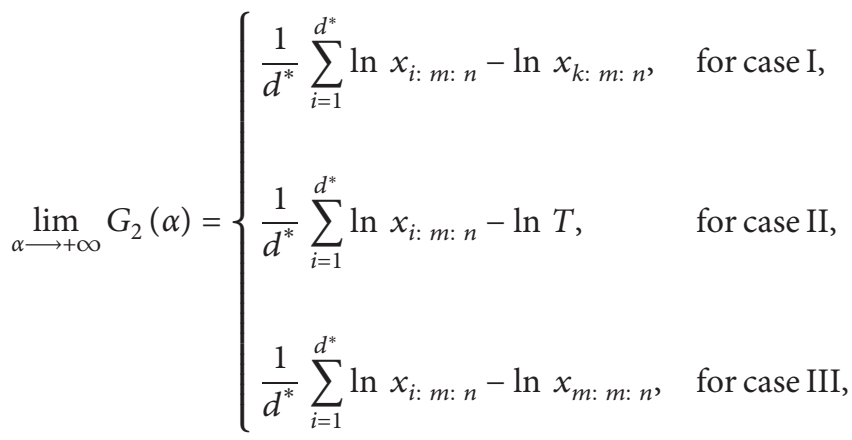

which indicates that the curves of $G_{1}(\alpha)$ and $G_{2}(\alpha)$ have a unique intersection point; therefore, the MLE of $\alpha$, as the root of equation $G_{1}(\alpha)=G_{2}(\alpha)$, exists and is unique. The result is proved.

\section{Data Availability}

The ball bearings failure data used to support the real-life illustration in the article are from a monograph, which has been cited. Other data used have also been included within the article.

\section{Conflicts of Interest}

The authors declare that there are no conflicts of interest regarding the publication of this paper.

\section{Acknowledgments}

This work of Liang Wang was supported by the National Natural Science Foundation of China (No. 12061091), the Yunnan Fundamental Research Project (No. 202101AT070103), and the Doctoral Research Foundation of Yunnan Normal University (No. 00800205020503129). This work of Xuanjia Zuo was supported by the Postgraduate Innovation Research Funding of Yunnan Normal University (No. YJSJJ21-B36).

\section{Supplementary Materials}

As complementary, the $\mathrm{R}$ code programme of the paper is provided in a separate supplementary file. (Supplementary Materials)

\section{References}

[1] W. Yan, P. Li, and Y. Yu, "Statistical inference for the reliability of Burr-XII distribution under improved adaptive Type-II progressive censoring," Applied Mathematical Modelling, vol. 95, pp. 38-52, 2021.

[2] B. Laumen and E. Cramer, "Progressive censoring with fixed censoring times," Statistics, vol. 53, no. 3, pp. 569-600, 2019.

[3] S. Gunasekera, "Inference for the Burr XII reliability under progressive censoring with random removals," Mathematics and Computers in Simulation, vol. 144, pp. 182-195, 2018.

[4] R. K. Maurya and Y. M. Tripathi, "Reliability estimation in a multicomponent stress-strength model for Burr XII distribution under progressive censoring," Brazilian Journal of Probability and Statistics, vol. 34, no. 2, pp. 345-369, 2020.

[5] N. Balakrishnan and E. Cramer, The Art of Progressive Censoring: Applications to Reliability and Quality, Birkhauser, New York, NY, USA, 2014.

[6] P. S. D. Mitra and D. Kundu, "A new decision theoretic sampling plan for type-i and type-i hybrid censored samples from the exponential distribution," Sankhya B, vol. 81, pp. 251-288, 2019.

[7] A. Algarni and A. M. Almarashi, "E-Bayesian estimation of chen distribution based on Type-I censoring scheme," Entropy, vol. 22, no. 6, p. 636, 2020.

[8] S. Basu, S. K. Singh, and U. Singh, "Bayesian inference using product of spacings function for progressive hybrid Type-I censoring scheme," Statistics, vol. 52, no. 2, pp. 345-363, 2018.

[9] E. Cramer, "Modularization of hybrid censoring schemes and its application to unified progressive hybrid censoring," Metrika, vol. 81, pp. 173-210, 2018.

[10] D. Singh, C. Lodhi, Y. M. Tripathi et al., "Inference for twoparameter Rayleigh competing risks data under generalized progressive hybrid censoring," Quality and Reliability Engineering International, vol. 37, no. 3, pp. 1210-1231, 2021. 
[11] N. Balakrishnan and D. Kundu, "Hybrid censoring models, inferential results and applications," Computational Statistics \& Data Analysis, vol. 57, pp. 166-209, 2013.

[12] Y. Cho, H. Sun, and K. Lee, "Exact likelihood inference for an exponential parameter under generalized progressive hybrid censoring scheme," Statistical Methodology, vol. 23, pp. 18-34, 2015.

[13] E. M. Almetwally and H. M. Almongy, "Maximum product spacing and bayesian method for parameter estimation for generalized power weibull distribution under censoring scheme," Journal of Data Science, vol. 17, no. 2, pp. 407-444, 2019.

[14] S. Chen, C. Qiao, Q. Ye et al., "Comparative study on threedimensional statistical damage constitutive modified model of rock based on power function and Weibull distribution," Environmental earth sciences, vol. 77, no. 108, pp. 1-16, 2018.

[15] A. Kumar and M. Ram, "System reliability analysis based on Weibull distribution and hesitant fuzzy set," International Journal of Mathematical, Engineering and Management Sciences, vol. 3, no. 4, pp. 513-521, 2018.

[16] A. Gelman, J. B. Carlin, H. S. Stern et al., Bayesian Data Analysis, Chapman and Hall/CRC, London, UK, 1995.

[17] L. Devroye, "A simple algorithm for generating random variates with a log-concave density," Computing, vol. 33, pp. 247-257, 1984.

[18] W. R. Gilks and P. Wild, "Adaptive rejection sampling for gibbs sampling," Applied Statistics, vol. 41, no. 2, pp. 337-348, 1992.

[19] N. Balakrishnan and R. Aggarwala, Progressive Censoring: Theory, Methods, Applications, Birkhauser, Boston, MA, USA, 2000.

[20] J. L. Lawless, Statistical Models and Methods for Life Time Data, Wiley, New York, NY, USA, 2ed. edition, 2003.

[21] H. M. Lee, W. C. Lee, C. L. Lei et al., "Computational procedure of assessing lifetime performance index of Weibull lifetime products with the upper record values," Mathematics and Computers in Simulation, vol. 81, no. 6, pp. 1177-1189, 2011. 